\title{
Method of DNA Extraction from Pinus rigida Wood Pretreated with Sandpaper ${ }^{1}$
}

\author{
Jamin Lee ${ }^{2} \cdot$ Tae-Jong Kim $\mathbb{D}^{2, \dagger}$
}

\begin{abstract}
Species identification of wood provides important information for archaeology, restoration of cultural assets, preventing illegal logging, and more. Wood species are usually identified based on their anatomical features with the use of a microscope. However, this method may not be able to distinguish between anatomically similar species or subspecies. To overcome this problem, wood species need to be identified at the molecular level using DNA sequencing. However, unlike living plant cells, wood is difficult to pulverize using a mortar, and DNA extraction from dried wood is challenging. To solve these problems, we propose a pretreatment method in which wood is pulverized using 60-grit sandpaper and hydrated with water for 2 days. Using this method, we were able to stably amplify the $r p o B$ gene from the extracted DNA of Pinus rigida. In addition, sequence analysis of the $r p o B$ gene revealed six single nucleotide polymorphisms (SNPs), which classified the $r р о B$ sequences in the genus Pinus into five groups. Our data indicate that although these SNPs were not suitable for species identification, they can potentially be used to determine the origin of different wood subspecies or individual samples of wood.
\end{abstract}

Keywords: species identification, sandpaper, hydration, rpoB, Pinus rigida

\section{INTRODUCTION}

To ensure the appropriate utilization of the designated species and to obtain important biological species information relevant to the restoration of cultural properties, archaeology, and forensic science, the accurate identification of wood species is important (Dumolin-Lapegue et al., 1999; Deguilloux et al., 2003; Rachmayanti et al., 2009). The identification of wood species is recognized as an important method for solving cases of illegal timber logging to protect forests (Rachmayanti et al., 2006; Dormontt et al., 2015). Most wood species have been identified successfully by anatomical observations using a microscope (Eom and Park, 2018; Kim and Choi, 2016; Kwon et al., 2017). However, the microscopic identification of wood species requires trained and experienced professionals to compare the anatomical features of different wood species (Wheeler et al., 1989; Wheeler and Baas, 1998; Ogata et al., 2008; Rachmayanti et al., 2009). In addition, the methods of anatomical identification of wood have limitations when specimens are structurally similar to each other, such as closely related species or subspecies (Marco et al., 1994; Feuillat et al., 1997;

\footnotetext{
${ }^{1}$ Date Received May 23, 2018, Date Accepted July 9, 2018

2 Department of Forest Products and Biotechnology, Kookmin University, Seoul 02707, Republic of Korea

† Corresponding author: Tae-Jong Kim (e-mail: bigbell@kookmin.ac.kr, ORCID: 0000-0002-7483-0432)
} 
Gasson, 2011; Jiao et al., 2015). To overcome these drawbacks, DNA sequencing is used as an alternative method of wood identification (Dumolin-Lapegue et al., 1999; Jiao et al., 2015). DNA sequencing enables the identification of wood at the molecular level and facilitates distinction between closely related species (Hebert et al., 2003; Hardy et al., 2006; Linacre and Tobe, 2011; Degen et al., 2013). Living plant cells actively use and maintain DNA; therefore, it is easy to extract and sequence DNA from living cells. In contrast, wood cells die after harvesting, resulting in fragmentation of DNA (Bär et al., 1988; Lindahl, 1993; Cano, 1996; Deguilloux et al., 2002; Pääbo et al., 2004; Rachmayanti et al., 2009). Therefore, it is difficult to extract DNA from wood. In addition, the methods used to extract DNA from living plant cells cannot be applied to wood because wood is subjected to a drying process to maintain its quality. For these reasons, the molecular identification of wood species has not been widely used, although it provides more precise information. These limitations hamper the establishment of a DNA database for the identification of wood species further.

To identify wood species using DNA sequencing, appropriate DNA markers must be selected. An ideal DNA marker is easily amplified from the fragmented DNA in wood samples and is able to simultaneously distinguish the samples. (Budowle and van Daal, 2008; Finkeldey et al., 2010). To meet these criteria, the chloroplast genome, existing in multiple copies in a single plant cell, is used for species identification (Deguilloux et al., 2002, 2003; Gailing et al., 2003; Indrioko et al., 2006). Single nucleotide polymorphisms (SNPs) in rpoB have been proposed for species identification in plants (Al-Qurainy et al., 2011; Khan et al., 2012). In this study, our purpose was to develop and validate a pretreatment method for extracting DNA from dried wood by hydration using water and pulverization using sandpaper. No special pretreatment method for extracting DNA from wood has been proposed previously. Based on our pretreatment method, we identified dried pitch pine using the extracted chloroplast gene $r p o B$, which encodes the $\beta$-subunit of RNA polymerase (National Center for Biotechnology Information accession number: JN854163.1), as a DNA marker. It is difficult to introduce random mutations in the conserved region because of its biological function; however, SNPs can be observed at nonconserved regions.

\section{MATERIALS and METHODS}

\subsection{Wood and Sandpaper}

Logs of sapwood $(5 \mathrm{~cm} \times 2.5 \mathrm{~cm} \times 1.5 \mathrm{~cm})$ of Pinus rigida were harvested in 2014. Six types of sandpaper (Chunil Grinding Co., Ltd., Seoul, Korea) with different roughness (40,50, 60, 80, 100, and 220 grit) were used.

\subsection{Preparation and Hydration of Wood Powder}

The wood specimens were autoclaved at $121^{\circ} \mathrm{C}$ for $20 \mathrm{~min}$ to eliminate any contamination. To remove surface contaminants further, a layer of approximately 1-mm thickness was removed from the surface of the wood specimens using sterilized sandpaper. Sandpaper with varying degrees of roughness was used to pulverize the specimens, and an optical microscope (Axio Imager.A1, Carl Zeiss Vision Korea Co., Ltd., Seoul, Korea) was used to observe the particle size of the powder. The wood powder $(20 \mathrm{mg}$ ) was collected in a sterilized centrifuge tube, and $200 \mu \mathrm{L}$ of sterilized distilled water was added as a hydration solvent. To suppress microbial growth, the mixtures of wood powder and water were incubated at $4^{\circ} \mathrm{C}$. 


\subsection{Extraction of DNA from Hydrated Wood Powder}

DNA was extracted from the hydrated wood powder with a DNeasy Blood \& Tissue Kit (catalog number: 69504; Qiagen Korea, Ltd., Seoul, Korea) according to the manufacturer's instructions. Briefly, $600 \mu \mathrm{L}$ of AP1 buffer and $6 \mu \mathrm{L}$ of RNase A (100 mg/mL) were added to the hydrated wood powder and mixed thoroughly. The sample was incubated at $65^{\circ} \mathrm{C}$ for 10 min and was inverted every 2 min for mixing. After incubation, $260 \mu \mathrm{L}$ of P3 buffer was added, and the sample was mixed well by inverting the tube. The fully mixed sample was placed in ice for $5 \mathrm{~min}$ and was centrifuged at 13,500 rpm for $10 \mathrm{~min}$. The supernatant was removed using a pipette tip with a truncated end and was transferred to a QIAshredder Mini Spin Column (Qiagen Korea, Ltd.). After centrifugation at 13,500 rpm for $2 \mathrm{~min}$, the solution was added to a new centrifuge tube. For each sample, a 1.5-fold volume of AW1 buffer was added to the solution, and the sample was immediately mixed using a pipette tip with a truncated end. The mixture (650 $\mu \mathrm{L})$ was added to the DNeasy Mini Spin Column (Qiagen Korea, Ltd.) and was centrifuged at 8,000 rpm for $1 \mathrm{~min}$. The flow-through solution was discarded, and the remaining mixture was added to the same column and was centrifuged at 8,000 rpm for $1 \mathrm{~min}$. The column was placed in a new collection tube, and $500 \mu \mathrm{L}$ of AW2 buffer was added to the column and was centrifuged at $8,000 \mathrm{rpm}$ for $1 \mathrm{~min}$. After the flow-through solution was discarded, the collection tube was remounted, and $500 \mu \mathrm{L}$ of AW2 buffer was added again and centrifuged at 13,500 rpm for $2 \mathrm{~min}$. The flow-through solution and the collection tube were discarded. The column was moved to a new centrifuge tube and was covered with clean tissue paper (KIMTECH, YuHan-Kimberly, Ltd., Seoul, Korea). The column was dried at room temperature for $40 \mathrm{~min}$. Subsequently, $50 \mu \mathrm{L}$ of AE buffer was placed in the center of the column, was incubated at room temperature for $5 \mathrm{~min}$, and then was centrifuged at 8,000 rpm for $1 \mathrm{~min}$. The DNA suspension obtained was stored in a freezer at $-20^{\circ} \mathrm{C}$.

\subsection{Amplification of $r p o B$ by Polymerase Chain Reaction (PCR)}

A 174-bp fragment of the $r p o B$ gene was amplified by PCR using the primers RPOB-1F (5-GCTTACACGA GCCCATATCC-3) and RPOB-1R (5-GGGATTT ACAGAATCGTGGTG-3) (Sun and Feng, 2011). PCR was performed in a $20-\mu \mathrm{L}$ volume containing $2 \mu \mathrm{L}$ of 10X Taq reaction buffer, $0.4 \mu \mathrm{L}$ of $10 \mathrm{mM} \mathrm{dNTP}$ mixture, $0.8 \mu \mathrm{L}$ of each $10 \mathrm{pM}$ primer, $0.1 \mu \mathrm{L}$ of

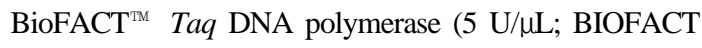
Co., Ltd., Daejeon, Korea), $2 \mu \mathrm{L}$ of extracted DNA template, and $13.9 \mu \mathrm{L}$ of water using GenePro Thermal Cycler (TC-E-48D; Hangzhou Bioer Technology Co., Ltd., Hangzhou, China). The PCR conditions were as follows: initial denaturation at $94^{\circ} \mathrm{C}$ for $5 \mathrm{~min}$, followed by 40 cycles of denaturation at $94^{\circ} \mathrm{C}$ for $1 \mathrm{~min}$, annealing at $55^{\circ} \mathrm{C}$ for $1 \mathrm{~min}$, and extension at $72^{\circ} \mathrm{C}$ for $30 \mathrm{~s}$, and a final extension at $72^{\circ} \mathrm{C}$ for $5 \mathrm{~min}$.

\subsection{Isolation and Purification of Amplified $r p o B$}

Two microliters of the $r p o B$ gene amplified by PCR were separated by gel electrophoresis using 1.5\% agarose gel. For gel extraction, buffer from the QIAquick Gel Extraction Kit (catalog number: 28706; Qiagen Korea, Ltd.) and columns from the HiGene ${ }^{\mathrm{TM}}$ Gel \& PCR Purification System (catalog number: GP104-100; BIOFACT Co., Ltd.) were used, and the DNA sample was extracted from the gel according to the instructions of the QIAquick Gel Extraction Kit. The purified DNA was confirmed by gel electrophoresis using $1.5 \%$ agarose gel. 


\subsection{DNA Sequence Analysis}

Purified PCR products were bidirectionally sequenced by BIOFACT Co., Ltd. A 133-nucleotide sequence was obtained (excluding the primer sequences) and was used as a query to search the nucleotide database of the National Center for Biotechnology Information (NCBI; https://www.ncbi.nlm.nih.gov/) using the nucleotide BLAST algorithm. The COBALT program available at the NCBI website was used to align all the similar sequences.

\section{RESULTS and DISCUSSION}

\subsection{Powdering Wood Using Sandpaper}

To extract DNA from a biological sample, cells must be ruptured. Living plant cells are surrounded by cell walls and membranes, which must be broken by enzymatic and physical methods. Dried wood is sturdy enough to withstand physical treatment. Therefore, methods used to break living plant cells are not applicable to wood. In this study, we used sandpaper with various degrees of roughness to pulverize the wood and break open the cells.

A microscope was used to observe the particle size of the wood powder obtained from sandpaper treatment (Fig. 1). The particle size of the wood powder decreased with increase in the grit number, a measure of the roughness of the sandpaper. With 60-grit sandpaper, wood particles of around $100 \mu \mathrm{m}$ in diameter were obtained. Because plant cells vary in size from 10 to $100 \mu \mathrm{m}$ (Smith, 2017), our results indicate that sandpaper with grit numbers of 60 or higher can break cells in wood samples. The $r p o B$ gene was successfully amplified six times from template DNA isolated from wood powder that was obtained using 60-grit sandpaper and was hydrated for 2 to 3 days.

This sandpaper method does not require special material or equipment, except for sandpaper, which can be easily purchased at low cost. In addition, because sandpaper is cheap, it can be used once for each sample, which prevents contamination of samples and facilitates the treatment of numerous samples in a relatively short time. Using sandpaper for pulverizing wood does not require special skills.
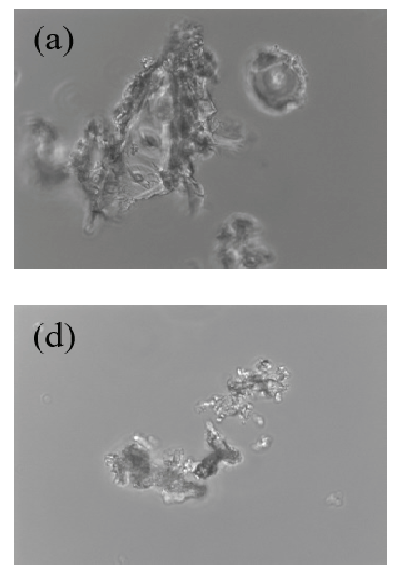
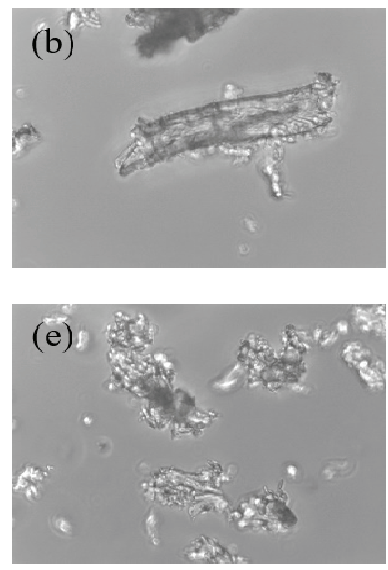
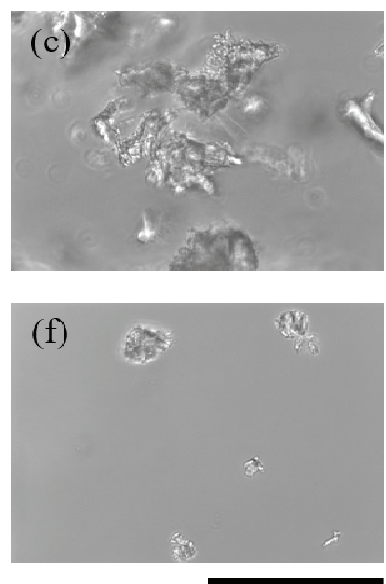

Fig. 1. Pulverizing the wood of Pinus rigida using sandpaper. Wood samples were pulverized using sandpaper of different degrees of roughness: 40 grit (a), 50 grit (b), 60 grit (c), 80 grit (d), 100 grit (e), and 220 grit (f). The images of wood particles were obtained using a microscope at $400 \times$ magnification. Scale bar: $100 \mu \mathrm{m}$. 


\subsection{Effect of Hydration Time on DNA Extraction}

DNA in the wood is fragmented and partially degraded and possibly sticks to the internal structure of the cell during the drying process (Rachmayanti et al., 2009). Even if the cell's structural integrity is destroyed by the sandpaper, the attached DNA cannot be eluted by a general DNA extraction method. In this study, we used a hydration process to elute the attached DNA. We determined the hydration time that was most effective for isolating DNA from wood powder.

To isolate DNA, wood powder obtained with the use of sandpaper was hydrated with distilled water for 1 to 5 days. The $r p o B$ gene was amplified from the DNA isolated from hydrated wood powder using PCR (Fig. 2). No amplification was obtained from DNA samples hydrated for 1 day, regardless of the roughness of the sandpaper. Amplification of the $r p o B$ gene was successfully observed in samples hydrated for 2 days or more. However, when the hydration period was 4 days or longer, the amplification success rate decreased. This observation supports the hypothesis of this study that the DNA in wood cells attaches to cell structures during the drying process. The decrease in the PCR

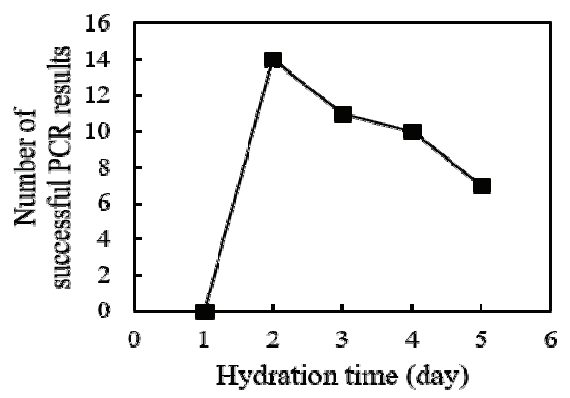

Fig. 2. Effect of hydration time of wood powder on polymerase chain reaction (PCR). The number of successful PCRs of the $r p o B$ gene ( $\mathrm{Y}$ axis) is shown as a function of the DNA extracted from wood powder hydrated for 1 to 5 days (X axis). Eighteen independent hydration experiments were conducted. success rate with prolonged hydration suggests that the eluted DNA may be degraded by contaminated enzymes or microorganisms during the long incubation period, even when the wood powder is hydrated at $4^{\circ} \mathrm{C}$. Overall, our data indicate that the optimal hydration time of wood powder for DNA elution is 2 days; this hydration time minimizes the degradation of eluted DNA while obtaining a sufficient DNA yield.

\subsection{Identification of SNPs of $r p o B$ in P. rigida}

In previous studies, intergenic spacer DNA sequences, psbA-trnH (Hong et al., 2014), atpF-atpH (Hong et al., 2014), and trnT-trnL (Um et al., 2014), in the chloroplast were used for taxonomic studies of the genus Pinus. In this study, SNPs of the $r p o B$ gene, which are used in classification of many plants, including the genus Pinus (Al-Qurainy et al., 2011; Khan et al., 2012), were analyzed for the evaluation of both the usefulness of $r p o B$ for identification of $P$. rigida and the efficiency of the pulverization and hydration pretreatment for DNA extraction. A 174-bp fragment of the $r p o B$ gene encoding the $\beta$-subunit of the chloroplast RNA polymerase was amplified (Fig. 3). The length of the PCR product was $174 \mathrm{bp}$ including the primer sequences and $133 \mathrm{bp}$ excluding the primer

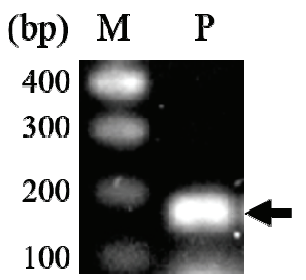

Fig. 3. Polymerase chain reaction (PCR) amplification of the $r p o B$ gene from DNA extracted using the method developed in this study. The amplified $r p o B$ gene was separated by gel electrophoresis using $1.5 \%$ agarose. The arrow on the right side indicates the amplified $r p o B$ gene fragment in lane P. M: marker. 


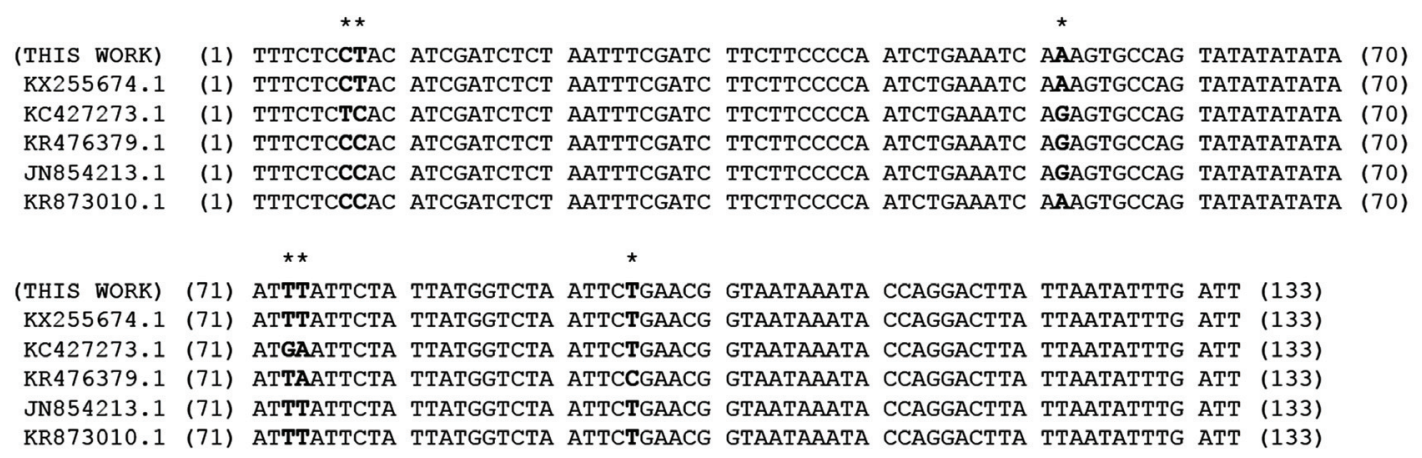

Fig. 4. Multiple sequence alignment of the $r p o B$ gene of $P$. rigida obtained in this study with $r p o B$ sequences of the genus Pinus. Nucleotides in bold with asterisks above the alignment indicate single nucleotide polymorphisms (SNPs).

sequences (Fig. 4 and Supplementary Fig. 1). The nucleotide sequence of the amplified $r p o B$ gene was used to search for similar sequences in the Pinus genus in the NCBI nucleotide database. Sequence analysis revealed SNPs at six locations in the gene sequence, and five groups were observed (Supplementary Fig. 1). The nucleotide sequences of representative genes of each group were aligned with those of $r p o B$ obtained in this study (Fig. 4). The nucleotide sequence of $r p o B$ in $P$. rigida was identical to that of the group containing $P$. koraiensis rpoB (NCBI accession number: AY228468). Five of the six SNPs differed from $P$. rigida rpoB (NCBI accession number: JN854163), which was reported previously in the NCBI nucleotide database as belonging to the second group. These results suggest that SNPs in the $r p o B$ gene, as identified previously (Sun and Feng, 2011) and analyzed in this study, may not be suitable for species identification; however, these SNPs can be used to determine the origin of different wood subspecies or individual samples of wood.

\section{CONCLUSION}

In this study, we propose a pretreatment method for wood samples that involves pulverizing the wood samples using 60-grit sandpaper followed by hydration with water for 2 days for DNA extraction. Pulverization of wood using sandpaper is inexpensive, requires no special equipment or skills, and eliminates the chance of contamination. DNA isolated by this method was a good template to amplify the $r p o B$ gene. Sequence analysis revealed five groups of SNPs in the $r p o B$ gene in the genus Pinus. Although these SNPs were not suitable for species identification, they can potentially be used to determine the origin of different wood subspecies or individual samples of wood.

\section{REFERENCES}

Al-Qurainy, F., Khan, S., Tarroum, M., Al-Hemaid, F.M., Ali, M.A. 2011. Molecular authentication of the medicinal herb Ruta graveolens (Rutaceae) and an adulterant using nuclear and chloroplast DNA markers. Genetics and Molecular Research 10(4): 2806-2816.

Bär, W., Kratzer, A., Mächler, M., Schmid, W. 1988. Postmortem stability of DNA. Forensic Science International 39(1): 59-70.

Budowle, B., van Daal, A. 2008. Forensically relevant SNP classes. Biotechniques 44(5): 603-608, 610.

Cano, R.J. 1996. Analysing ancient DNA. Endeavour 20(4): 162-167. 
Degen, B., Ward, S.E., Lemes, M.R., Navarro, C., Cavers, S., Sebbenn, A.M. 2013. Verifying the geographic origin of mahogany (Swietenia macrophylla King) with DNA-fingerprints. Forensic Science InternationalGenetics 7(1): 55-62.

Deguilloux, M.F., Pemonge, M.H., Petit, R.J. 2002. Novel perspectives in wood certification and forensics: dry wood as a source of DNA. Proceedings Biological Sciences 269(1495): 1039-1046.

Deguilloux, M.F., Pemonge, M.H., Bertel, L., Kremer, A., Petit, R.J. 2003. Checking the geographical origin of oak wood: molecular and statistical tools. Molecular Ecology 12(6): 1629-1636.

Dormontt, E.E., Boner, M., Braun, B., Breulmann, G., Degen, B., Espinoza, E., Gardner, S., Guillery, P., Hermanson, J.C., Koch, G., Lee, S.L., Kanashiro, M., Rimbawanto, A., Thomas, D., Wiedenhoeft, A.C., Yin, Y.F., Zahnen, J., Lowe, A.J. 2015. Forensic timber identification: It's time to integrate disciplines to combat illegal logging. Biological Conservation 191: 790-798.

Dumolin-Lapegue, S., Pemonge, M.H., Gielly, L., Taberlet, P., Petit, R.J. 1999. Amplification of oak DNA from ancient and modern wood. Molecular Ecology 8(12): 2137-2140.

Eom, Y.-J., Park, B.-D. 2018 Wood species identification of documentary woodblocks of Songok clan of the Milseong Park, Gyeongju, Korea. Journal of the Korean Wood Science and Technology 46(3): 270-277.

Feuillat, F., Dupouey, J.L., Sciama, D., Keller, R. 1997. A new attempt at discrimination between Quercus petraea and Quercus robur based on wood anatomy. Canadian Journal of Forest Research-Revue Canadienne De Recherche Forestiere 27(3): 343-351.

Finkeldey, R., Leinemann, L., Gailing, O. 2010. Molecular genetic tools to infer the origin of forest plants and wood. Applied Microbiology and Biotechnology 85(5): 1251-1258.
Gailing, O., Wachter, H., Leinemann, L., Hosius, B., Finkeldey, R., Schmitt, H.P., Heyder, J. 2003. Characterisation of different provenances of late flushing pedunculate oak (Quercus robur L.) with chloroplast markers. Allgemeine Forst Und Jagdzeitung 174(12): 227-231.

Gasson, P. 2011. How precise can wood identification be? Wood anatomy's role in support of the legal timber trade, especially cites. IAWA Journal 32(2): 137-154.

Hardy, O.J., Maggia, L., Bandou, E., Breyne, P., Caron, H., Chevallier, M.H., Doligez, A., Dutech, C., Kremer, A., Latouche-Halle, C., Troispoux, V., Veron, V., Degen, B. 2006. Fine-scale genetic structure and gene dispersal inferences in 10 neotropical tree species. Molecular Ecology 15(2): 559-571.

Hebert, P.D.N., Cywinska, A., Ball, S.L., DeWaard, J.R. 2003. Biological identifications through DNA barcodes. Proceedings of the Royal Society B-Biological Sciences 270(1512): 313-321.

Hong, J.-K., Yang, J.-C., Lee, Y.-M., Kim, J. H. 2014. Molecular phylogenetic study of Pinus in Korea based on chloroplast DNA psbA-trnH and atpF-H sequences data. Korean Journal of Plant Taxonomy 44(2): 111-118.

Indrioko, S., Gailing, O., Finkeldey, R. 2006. Molecular phylogeny of Dipterocarpaceae in Indonesia based on chloroplast DNA. Plant Systematics and Evolution 261(1-4): 99-115.

Jiao, L.C., Liu, X.L., Jiang, X.N., Yin, Y.F. 2015. Extraction and amplification of DNA from aged and archaeological Populus euphratica wood for species identification. Holzforschung 69(8): 925931.

Khan, S., Al-Qurainy, F., Nadeem, M., Tarroum, M. 2012. Development of genetic markers for Ochradenus arabicus (Resedaceae), an endemic medicinal plant of Saudi Arabia. Genetics and Molecular Research 11(2): 1300-1308. 
Kim, S.C., Choi, J. 2016. Study on wood species identification for Daeungjeon hall of Jeonghyesa temple, Suncheon. Journal of the Korean Wood Science and Technology 44(6): 897-902.

Kwon, O., Lee, H.G., Lee, M.-R., Jang, S., Yang, S.-Y., Park, S.-Y., Choi, I.-G., Yeo, H. 2017. Automatic wood species identification of Korean softwood based on convolutional neural networks. Journal of the Korean Wood Science and Technology 45(6): 797-808.

Linacre, A., Tobe, S.S. 2011. An overview to the investigative approach to species testing in wildlife forensic science. Investigative Genetics 2(1): 2.

Lindahl, T. 1993. Instability and decay of the primary structure of DNA. Nature 362(6422): 709-715.

Marco, J., Artajona, J., Larrechi, M.S., Rius, F.X. 1994. Relationship between geographical origin and chemical-composition of wood for oak barrels. American Journal of Enology and Viticulture 45(2): 192-200.

Ogata, K., Fujii, T., Abe, H., Baas, P. 2008. Identification of the timbers of Southeast Asia and the Western Pacific. Kaiseisha Press, Otsu, Japan. Pääbo, S., Poinar, H., Serre, D., Jaenicke-Després, V., Hebler, J., Rohland, N., Kuch, M., Krause, J., Vigilant, L., Hofreiter, M. 2004. Genetic analyses from ancient DNA. Annual Review of Genetics 38: 645-679.
Rachmayanti, Y., Leinemann, L., Gailing, O., Finkeldey, R. 2006. Extraction, amplification and characterization of wood DNA from Dipterocarpaceae. Plant Molecular Biology Reporter 24(1): 45-55.

Rachmayanti, Y., Leinemann, L., Gailing, O., Finkeldey, R. 2009. DNA from processed and unprocessed wood: Factors influencing the isolation success. Forensic Science International: Genetics 3(3): 185192.

Smith, D.R. 2017. Does cell size impact chloroplast genome size? Frontiers in Plant Science 8(2116): 1-6.

Sun, X., Feng, F. 2011. Development and analysis on microsatellite sequence of chloroplast DNA of Pinus koraiensis. 2011 5th International Conference on Bioinformatics and Biomedical Engineering, Wuhan, China.

Um, Y., Park, W.-K., Jo, N.-S., Han, S.-H., Lee, Y. 2014. Phylogenetic analysis of pines based on chloroplast trnT-trnL intergenic spacer DNA sequences. Journal of Forest and Environmental Science 30(3): 307-313.

Wheeler, E., Baas, P., Gasson, P. 1989. IAWA list of microcopie features for hardwood identification, National Herbarium of the Netherlands, Netherlands. Wheeler, E.A., Baas, P. 1998. Wood identification A review. IAWA Journal 19(3): 241-264. 
Supplementary Fig. 1. DNA sequence alignment of the rроB fragment from this work with 115 rpoB fragments of Pinus from the nucleotide database of the National Center for Biotechnology Information.

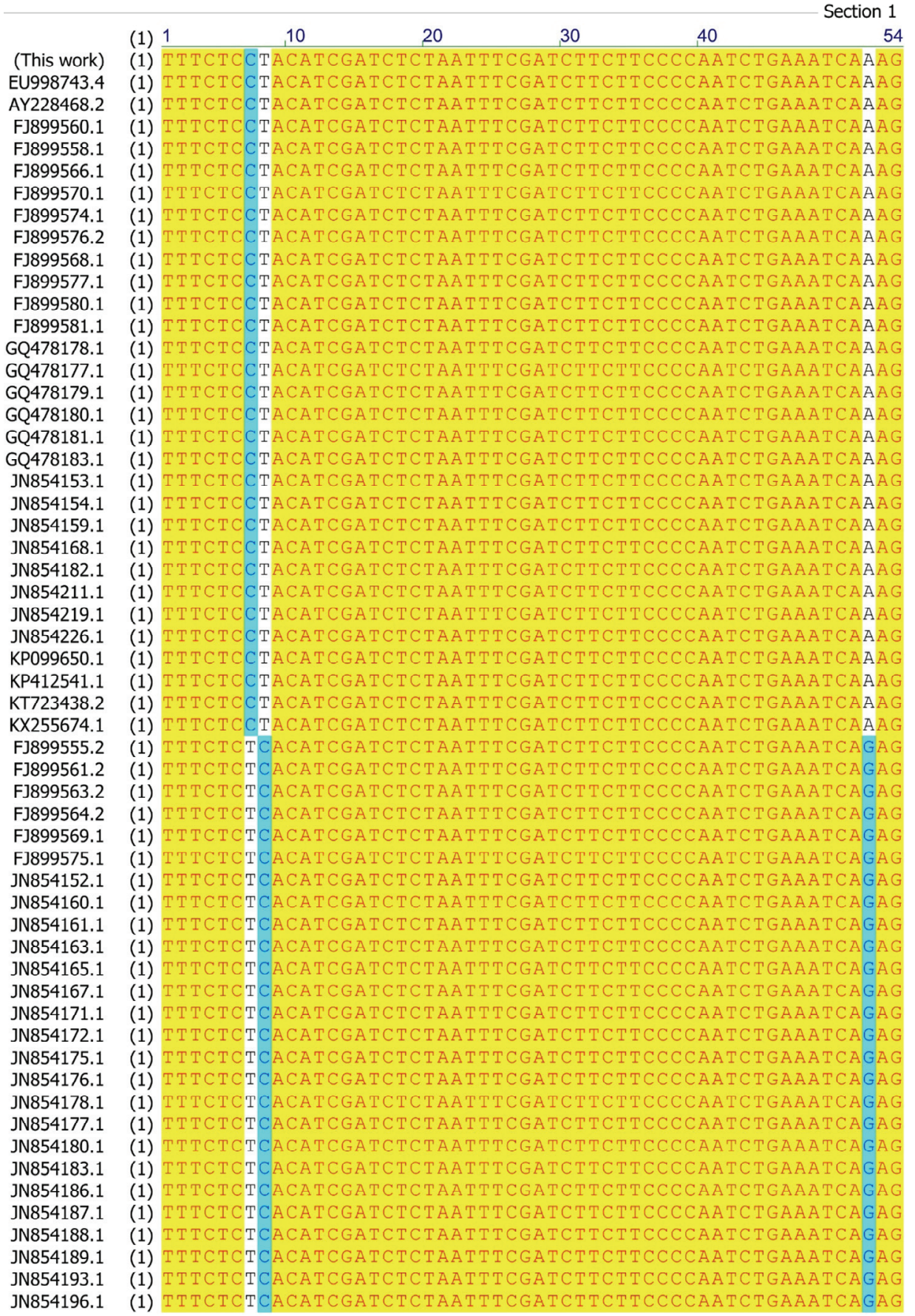


Method of DNA Extraction from Pinus rigida Wood Pretreated with Sandpaper
JN854198.1
(1) TTTCTCTCACATCGATCTCTAATTTCGACTTCTTCCCCAATCTGAAATCAGAG
JN854199.1
(1) тTтCTCTCACATCGATCTCTAAтTTCGATCTTCTTCCCCAAтCTGAAATCAGA
JN854201.1
JN854205.1
JN854202.1
JN854206.1
JN854208.1
(1) TTTCTCTCACATCGATCTCTAATTTCGATCTTCTTCCCCAATCTGAAATCAGAG
(1) TTTCTCTCACATCGATCTCTAATTCGATCTTCTTCCCCAATCTGAAATCAGAG
(1) TTTCTCTCACATCGATCTCTAATTCGATCTTCTTCCCCAATCTGAAATCAGAG
(1) TTTCTCTCACATCGATCTCTAATTTCGATCTTCTTCCCCAATCTGAAATCAGAG
JN854214.1
JN854215.1
(1) TTTCTCTCACATCGATCTCTAATTCGATCTTCTTCCCCAAтCTGAAATCAgAg

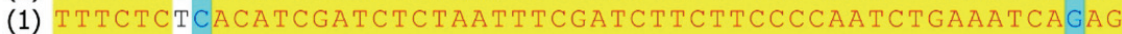
JN854216.1
JN854218.1
(1) TTTCTCTCACATCGATCTCTAATTTCGATCTTCTTCCCCAATCTGAAATCAGAG
(1) TTTCTCTCACATCGATCTCTAAтTCGATCTTCTTCCCCAATCTGAAATCAGAG
JN854222.1
(1) TTTCTCTCACATCGATCTCTAATTTCGATCTTCTTCCCCAATCTGAAATCAGAG
JN854225.1
(1) тTTCTCTCACATCGATCTCTAAтTCGATCTTCTTCCCCAAтCTGAAATCAgAg
KC427273.1

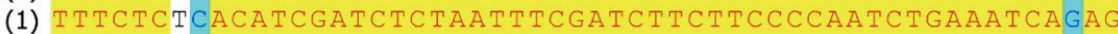
D17510.1
FJ899556.1
(1) TTTCTCTCACATCGATCTCTAATTCGATCTTCTTCCCCAATCTGAAATCAGAG
FJ899562.1
(1) TTTCTCCCACATCGATCTCTAATTCGATCTTCTTCCCCAATCTGAAATCAGAG
(1) TTTCTCCCACATCGATCTCTAATTTCGATCTTCTTCCCCAATCTGAAATCAGAG
FJ899572.2
FJ899579.1
(1) TTTCTCCCACATCGATCTCтAAтTCGATCTTCTTCCCCAAтCTGAAATCAGAG
(1) тTTCTCCCACATCGATCTCTAAтTCGATCTTCTTCCCCAATCTGAAATCAGAG
JN854151.1
JN854156.1
(1) тTTCTCCCACATCGATCTCTAATTTCGATCTTCTTCCCCAATCTGAAATCAGAG
(1) TTTCTCCCACATCGATCTCTAATTCGATCTTCTTCCCCAATCTGAAATCAGAG
JN854158.1
(1) TTTCTCCCACATCGATCTCTAATTTCGATCTTCTTCCCCAATCTGAAATCAGAG
JN854162.1
JN854173.1
JN854179.1
(1) TTTCTCCCACATCGATCTCTAAтTCGATCTTCTTCCCCAAтCTGAAATCAGAG
(1) TTTCTCCCACATCGATCTCTAATTTCGATCTTCTTCCCCAATCTGAAATCAGAG
JN854181.1
JN854185.1
(1) TTTCTCCCACATCGATCTCTAAтTTCGATCTTCTTCCCCAATCTGAAATCAGA
(1) TTTCTCCCACATCGATCTCTAATTTCGATCTTCTTCCCCAATCTGAAATCAGAG
(1) TTTCTCCCACATCGATCTCTAATTTCGATCTTCTTCCCCAATCTGAAATCAGAG
JN854190.1
JN854191.1
JN854194.1
JN854197.1
JN854200.1
JN854209.1
JN854210.1
JN854224.1
KC427272.1
KP771703.1
KR476379.1
KT740995.1
KX833097.1
EU998744.3
EU998745.4
EU998746.4
FJ899557.1
FJ899567.2
JN854164.1
JN854166.1
(1) TTTCTCCCACATCGATCTCTAAтT TGATCTTCTTCCCCAAтCTGAAATCAGAG
(1) TTTCTCCCACATCGATCTCTAATTTCGATCTTCTTCCCCAATCTGAAATCAGAG
(1) TTTCTCCCACATCGATCTCTAATTTCGATCTTCTTCCCCAATCTGAAATCAGAG
(1) TTTCTCCCACATCGATCTCTAAтTCGATCTTCTTCCCCAATCTGAAATCAGAG
(1) TTTCTCCCACATCGATCTCTAATTTCGATCTTCTTCCCCAATCTGAAATCAGAG
(1) TTTCTCCCACATCGATCTCTAATTCGATCTTCTTCCCCAATCTGAAATCAGAG
(1) TTTCTCCCACATCGATCTCTAATTTCGATCTTCTTCCCCAATCTGAAATCAGAG
(1) TTTCTCCCACATCGATCTCTAATTCGATCTTCTTCCCCAAтCTGAAATCAGAG
(1) TTTCTCCCACATCGATCTCTAATTTCGACTTCTTCCCCAATCTGAAATCAGAG
(1) TTTCTCCCACATCGATCTCTAATTTCGATCTTCTTCCCCAATCTGAAATCAGAG
(1) TTTCTCCCACATCGATCTCTAATTTCGATCTTCTTCCCCAATCTGAAATCAGAG
(1) TTTCTCCCACATCGATCTCTAATTTCGATCTTCTTCCCCAATCTGAAATCAGAG
(1) TTTCTCCCACATCGATCTCTAATTTCGATCTTCTTCCCCAATCTGAAATCAGAG
(1) TTTCTCCCACATCGATCTCTAATTCGATCTTCTTCCCCAATCTGAAATCAGAG
(1) TTTCTCCCACATCGATCTCTAATTTCGATCTTCTTCCCCAATCTGAAATCAGAG
(1) TTTCTCCCACATCGATCTCTAAтTTCGACTTCTTCCCCAATCTGAAATCAGAG
(1) TTTCTCCCACATCGATCTCTAATTTCGATCTTCTTCCCCAATCTGAAATCAGAG
(1) TTTCTCCCACATCGATCTCTAATTTCGATCTTCTTCCCCAATCTGAAATCAGAG
(1) TTTCTCCCACATCGATCTCTAATTTCGATCTTCTTCCCCAATCTGAAATCAGAG
(1) T T T TCCCACATCGATCTCTAAтT TCGATCTTCTTCCCCAATCTGAAATCAGAG
(1) TTTCTCCCACATCGATCTCTAATTCGATCTTCTTCCCCAATCTGAAATCAGAG
JN854174.1
JN854184.1
JN854192.1
JN854203.1
JN854207.1
(1) тTTCTCCCACATCGATCTCTAAтTCGATCTTCTTCCCCAATCTGAAATCAGAG
(1) TTTCTCCCACATCGATCTCTAATTTCGATCTTCTTCCCCAATCTGAAATCAGAG
(1) TTTCTCCCACATCGATCTCTAATTTCGATCTTCTTCCCCAATCTGAAATCAGAG
(1) TTTCTCCCACATCGATCTCTAATTTCGATCTTCTTCCCCAATCTGAAATCAGAG
JN854213.1
JN854220.1

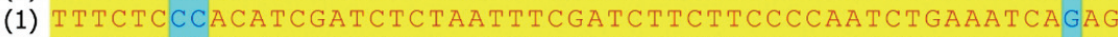
(1) TTTCTCCCACATCGATCTCTAATTTCGATCTTCTTCCCCAATCTGAAATCAGAG
EU998741.4
(1) TTTCTCCCACATCGATCTCTAAтTTCGATCTTCTCCCCAATCTGAAATCAGA
EU998742.4
FJ899559.1
(1) TTTCTCCCACATCGATCTCTAATTTCGATCTTCTTCCCCAATCTGAAATCAAAG
(1) TTTCTCCCACATCGATCTCTAATTTCGATCTTCTTCCCCAATCTGAAATCAAAG
(1) тTTCTCCCACATCGATCTCTAATTCGATCTTCTTCCCCAATCTGAAATCAAAG
JN854223.1
KR873010.1
(1) TTTCTCCCACATCGATCTCTAATTTCGATCTTCTTCCCCAATCTGAAATCAAAG
(1) TTTCTCCCACATCGATCTCTAATTCGATCTTCTTCCCCAATCTGAAATCAAAG 
Jamin Lee $\cdot$ Tae-Jong Kim

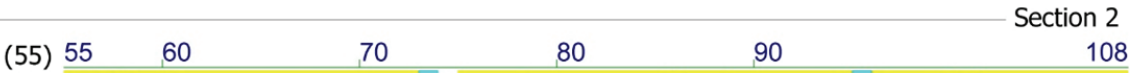

(This work) (55) TGCCAGTATATATATAATTT ATTCTATTATGGTCTAATTCTGAACGGTAAT A A EU998743.4 (55) TGCCAGTATATATATAATT TATTCTATTATGGTCTAAT TCTGAACG TAATAAA AY228468.2 (55) TGCCAGTATATATATAATTATTCTATTATGGTCTAATTCTGAACGGTAATAA FJ899560.1 (55) TGCCAGTATATATATAATTATTCTATTATGGTCTAATTCTGAACGGTAATAAA FJ899558.1 (55) TGCCAGTATATATATAATTATTCTATTATGGTCTAATCTGAACGGTAATAAA FJ899566.1 (55) TGCCAGTATATATATAATTATTCTATTATGGTCTAATTCTGAACGGTAATAA FJ899570.1 (55) TGCCAGTATATATATAATTATTCTATTATGGTCTAATTCTGAACGGTAATAAA FJ899574.1 (55) TGCCAGTATATATATAATTATTCTATTATGGTCTAATTCTGAACGGTAATAAA FJ899576.2 (55) TGCCAGTATATATATAATTATTCTATTATGGTCTAATTCTGAACGGTAATAAA FJ899568.1 (55) TGCCAGTATATAтAтAATTATTCTATTATGTCTAATTCTGAACGGTAAтAAA FJ899577.1 (55) TGCCAGTATATATATAATTATTCTATTATGGTCTAATTCTGAACGGTAATAAA FJ899580.1 (55) TGCCAGTATATATATAATTATTCTATATGGTCTAATTCTGAACGGTAATAA FJ899581.1 (55) TGCCAGTATATATATAATTATTCTATATGGTCTAATTCTGAACGGTAATAA GQ478178.1 (55) TGCCAGTATATATATAATTTATTCTATTATGGTCTAATTCTGAACGGTAATAAA GQ478177.1 (55) TGCCAGTATATATATAATTATTCTATTATGGTCTAATTCTGAACGGTAATAAA GQ478179.1 (55) TGCCAGTATATATATAATTATTCTATTATGGTCTAATTCTGAACGGTAATAAA GQ478180.1 (55) TGCCAGTATATATATAATTATTCTATTATGGTCTAATTCTGAACGGTAATAAA GQ478181.1 (55) TGCCAGTATATATATAATTATTCTATTATGGTCTAATTCTGAACGGTAATAAA GQ478183.1 (55) TGCCAGTATATATATAATTTATTCTATTATGGTCTAATTCTGAACGGTAATAAA JN854153.1 (55) TGCCAGTATATATATAATTATTCTATTATGGTCTAATTCTGAACGGTAATAAA JN854154.1 (55) TGCCAGTATATATATAATTATTCTATTATGGTCTAATTCTGAACGGTAATAAA JN854159.1 (55) TGCCAGTATATATATAATTATTCTATTATGGTCTAATTCTGAACGGTAATAAA JN854168.1 (55) TGCCAGTATATATATAATTATTCTATTATGGTCTAATTCTGAACGGTAATAAA JN854182.1 (55) TGCCAGTATATATATAATTTATTCTATTATGGTCTAATTCTGAACGGTAATAAA JN854211.1 (55) TGCCAGTATATATATAATTATTCTATTATGGTCTAATTCTGAACGGTAATAAA JN854219.1 (55) TGCCAGTATATATATAATTATTCTATTATGGTCTAATTCTGAACGGTAATAAA JN854226.1 (55) TGCCAGTATATATATAATTATTCTATTATGGTCTAATTCTGAACGGTAATAAA KP099650.1 (55) TGCCAGTATATATATAATT TATTCTATTATGGTCTAATTCTGAACGGTAATAAA KP412541.1 (55) TGCCAGTATATATATAATTTATTCTATTATGGTCTAAT TCTGAACGGTAATAAA KT723438.2 (55) TGCCAGTATATATATAATTATTCTATATGGTCTAATTCTGAACGGTAAAAA KX255674.1 (55) TGCCAGTATATATATAATT TATTCTATTATGGTCTAAT TCTGAACGGTAATAAA FJ899555.2 (55) TGCCAGTATATATATAATGAATCTATTATGGTCTAATTCTGAACGGTAATAAA FJ899561.2 (55) TGCCAGTATATATATAATGAATCTATTATGGTCTAATTCTGAACGGTAATAAA FJ899563.2 (55) TGCCAGTATATATATAATGAATCTATTATGGTCTAATTCTGAACGGTAATAAA FJ899564.2 (55) TGCCAGTATATATATAATGAATCTATTATGGTCTAATTCTGAACGGTAATAAA FJ899569.1 (55) TGCCAGTATATATATAATGAATTCTATTATGGTCTAATTCTGAACGGTAATAAA FJ899575.1 (55) TGCCAGTATATATATAATGAATCTATTATGGTCTAATTCTGAACGGTAATAAA JN854152.1 (55) TGCCAGTATATATATAATGAATCTATTATGGTCTAATTCTGAACGGTAATAAA JN854160.1 (55) TGCCAGTATATATATAATGAATCTATTATGGTCTAATTCTGAACGGTAATAAA JN854161.1 (55) TGCCAGTATATATATAATGAATCTATTATGGTCTAATTCTGAACGGTAATAAA JN854163.1 (55) TGCCAGTATATATATAATGAATCTATTATGGTCTAATTCTGAACGGTAATAAA JN854165.1 (55) TGCCAGTATATATATAATGAATCTATTATGGTCTAATTCTGAACGGTAATAAA JN854167.1 (55) TGCCAGTATATATATAATGAATCTATTATGGTCTAATTCTGAACGGTAATAAA JN854171.1 (55) TGCCAGTATATATATAATGAATCTATTATGGTCTAATTCTGAACGGTAATAAA JN854172.1 (55) TGCCAGTATATATATAATGAATCTATTATGGTCTAATTCTGAACGGTAATAAA JN854175.1 (55) TGCCAGTATATATATAATGAATCTATTATGGTCTAATTCTGAACGGTAATAAA JN854176.1 (55) TGCCAGTATATATATAATGAATCTATTATGGTCTAATTCTGAACGGTAATAAA JN854178.1 (55) TGCCAGTATATATATAATGAATCTATTATGGTCTAATTCTGAACGGTAATAAA JN854177.1 (55) TGCCAGTATATATATAATGAATCTATTATGGTCTAATTCTGAACGGTAATAAA JN854180.1 (55) TGCCAGTATATATATAATGAATCTATTATGGTCTAAT TCTGAACGGTAATAAA JN854183.1 (55) TGCCAGTATATATATAATGAATCTATTATGGTCTAATTCTGAACGGTAATAAA JN854186.1 (55) TGCCAGTATATATATAATGAATCTATTATGGTCTAATTCTGAACGGTAATAAA JN854187.1 (55) TGCCAGTATATATATAATGAATTCTATTATGGTCTAAT TCTGAACGGTAATAAA JN854188.1 (55) TGCCAGTATATATATAATGAATCTATTATGGTCTAATTCTGAACGGTAATAAA JN854189.1 (55) TGCCAGTATATATATAATGAATCTATTATGGTCTAATTCTGAACGGTAATAAA JN854193.1 (55) TGCCAGTATATATATAATGAATCTATTATGGTCTAATTCTGAACGGTAATAAA JN854196.1 (55) TGCCAGTATATATATAATGAATCTATTATGGTCTAATTCTGAACGGTAATAAA 
Method of DNA Extraction from Pinus rigida Wood Pretreated with Sandpaper

\begin{abstract}
JN854198.1 (55) TGCCAGTATATATATAATGAATCTATTATGGTCTAATTCTGAACGGTAATAAA JN854199.1 (55) TGCCAGTATATAтATAATGATTCTATTATGGTCTAATTCTGAACGGTAATAA JN854201.1 (55) TGCCAGTATATATATAATGAATCTATTATGGTCTAATTCTGAACGGTAATAAA JN854205.1 (55) TGCCAGTATATATATAATGAATCTATTATGGTCTAATTCTGAACGGTAATAAA JN854202.1 (55) TGCCAGTATATATATAATGAATCTATTATGGTCTAATTCTGAACGGTAATAAA JN854206.1 (55) TGCCAGTATATATATAATGAATTCTATTATGGTCTAATTCTGAACGGTAATAAA JN854208.1 (55) TGCCAGTATATAтATAAтAATTTATTATGGTCTAATTCTGAACGGTAAтAA JN854214.1 (55) TGCCAGTATATATATAATAATTCTATATGGTCTAATTCTGAACGGTAATAAA JN854215.1 (55) TGCCAGTATATATATAATGAATTCTATTATGGTCTAATTCTGAACGGTAATAAA JN854216.1 (55) TGCCAGTATATATATAATGAATCTATTATGGTCTAATTCTGAACGGTAATAAA JN854218.1 (55) TGCCAGTATATATATAATGAATTCTATATGGTCTAATTCTGAACGGTAATAAA JN854222.1 (55) TGCCAGTATATATATAATGAATCTATTATGGTCTAATTCTGAACGGTAATAAA JN854225.1 (55) TGCCAGTATATATATAATGAATCTATTATGGTCTAATTCTGAACGGTAATAAA KC427273.1 (55) TGCCAGTATATATATAATGATTCTATTATGGTCTAATTCTGAACGGTAATAAA

D17510.1 (55) TGCCAGTATATATATAATAATCTATtATGGTCTAATCCGAACGGTAATAA FJ899556.1 (55) TGCCAGTATATATATAATTAATCTATTATGGTCTAATTCCGAACGGTAATAAA FJ899562.1 (55) TGCCAGTATATATATAATAATCTATTATGGTCTAATTCCGAACGGTAAAAA FJ899572.2 (55) TGCCAGTATATATATAATAATTCATTATGGTCTAATTCCGAACGGTAATAAA FJ899579.1 (55) TGCCAGTATATATATAATAATTTATTATGGTCTAATTCCGAACGGTAATAAA JN854151.1 (55) TGCCAGTATATATATAATTAATCTATTATGGTCTAATTCCGAACGGTAATAAA JN854156.1 (55) TGCCAGTATATATATAATTAATTCTATTATGGTCTAATTCCGAACGGTAATAAA JN854158.1 (55) TGCCAGTATATATATAATTAATCTATTATGGTCTAATTCCGAACGGTAATAAA JN854162.1 (55) TGCCAGTATATATATAATTAATTCTATATGGTCTAATTCCGAACGGTAATAAA JN854173.1 (55) TGCCAGTATATATATAATAATCTATTATGGTCTAATCCGAACGGTAATAA JN854179.1 (55) TGCCAGTATATATATAATTAATTCTATTATGGTCTAATTCCGAACGGTAATAAA JN854181.1 (55) TGCCAGTATATATATAATAATTCTATTATGGTCTAATTCCGAACGGTAATAAA JN854185.1 (55) TGCCAGTATATATATAATAATCTATTATGGTCTAATTCCGAACGGTAATAAA JN854190.1 (55) TGCCAGTATATATATAATTAATTCTATTATGGTCTAATTCCGAACGGTAATAAA JN854191.1 (55) TGCCAGTATATATATAATTAATCTATTATGGTCTAATTCCGAACGGTAATAAA JN854194.1 (55) TGCCAGTATATATATAATTAATCTATTATGGTCTAATTCCGAACGGTAATAAA JN854197.1 (55) TGCCAGTATATATATAATTAATTCTATTATGGTCTAATTCCGAACGGTAATAAA JN854200.1 (55) TGCCAGTATATATATAATTAATCTATTATGGTCTAATTCCGAACGGTAATAAA JN854209.1 (55) TGCCAGTATATATATAATAATTCTATTATGGTCTAATTCCGAACGGTAATAAA JN854210.1 (55) TGCCAGTATATATATAATTAATTCTATTATGGTCTAATTCCGAACGGTAATAAA JN854224.1 (55) TGCCAGTATATATATAATAATTCTATTATGGTCTAATTCCGAACGGTAATAAA KC427272.1 (55) TGCCAGTATATATATAATTAAT TCTATTATGGTCTAATTCCGAACGGTAATAAA KP771703.1 (55) TGCCAGTATATATATAATTAATCTATTATGGTCTAATTCCGAACGGTAATAAA KR476379.1 (55) TGCCAGTATATATATAATTAATTCTATTATGGTCTAATTCCGAACGGTAATAAA KT740995.1 (55) TGCCAGTATATATATAATTAATCTATTATGGTCTAATTCCGAACGGTAATAAA KX833097.1 (55) TGCCAGTATATATATAATTAATTCATTATGGTCTAATTCCGAACGGTAATAAA EU998744.3 (55) TGCCAGTATATATATAATTATTCTATTATGGTCTAATTCTGAACGGTAATAAA EU998745.4 (55) TGCCAGTATATATATAATTATTCTATTATGGTCTAATTCTGAACGGTAATAAA EU998746.4 (55) TGCCAGTATATATATAATTATTCTATTATGGTCTAATTCTGAACGGTAATAAA FJ899557.1 (55) TGCCAGTATATATATAATTATTCTATTATGGTCTAATTCTGAACGGTAATAAA FJ899567.2 (55) TGCCAGTATATATATAATTATTCTATTATGGTCTAATTCTGAACGGTAATAAA JN854164.1 (55) TGCCAGTATATATATAATTATTCTATTATGGTCTAATTCTGAACGGTAATAAA JN854166.1 (55) TGCCAGTATATATATAATTAATCTATTATGGTCTAATTCTGAACGGTAATAAA JN854174.1 (55) TGCCAGTATATATATAATTATTCTATTATGGTCTAATTCTGAACGGTAATAAA JN854184.1 (55) TGCCAGTATATATATAATTATTCTATTATGGTCTAATTCTGAACGGTAATAAA JN854192.1 (55) TGCCAGTATATATATAATTATTCTATTATGGTCTAATTCTGAACGGTAATAAA JN854203.1 (55) TGCCAGTATATATATAATTATTCTATTATGGTCTAATTCTGAACGGTAATAAA JN854207.1 (55) TGCCAGTATATATATAATTATTCTATTATGGTCTAATTCTGAACGGTAATAAA JN854213.1 (55) TGCCAGTATATATATAATTATTCTATTATGGTCTAATTCTGAACGGTAATAAA JN854220.1 (55) TGCCAGTATATATATAATTATTCTATTATGGTCTAATTCTGAACGGTAATAAA EU998741.4 (55) TGCCAGTATATATATAATTATTCTATTATGGTCTAATTCTGAACGGTAATAAA EU998742.4 (55) TGCCAGTATATATATAATTTATTCTATTATGGTCTAATTCTGAACGGTAATAAA FJ899559.1 (55) TGCCAGTATATATATAATTATTCTATTATGGTCTAATTCTGAACGGTAATAAA JN854223.1 (55) TGCCAGTATATATATAATT TAT TCTATTATGGTCTAAT TCTGAACGGTAATAAA KR873010.1 (55) TGCCAGTATATATATAATTATTCTATTATGGTCTAATTCTGAACGGTAATAAA
\end{abstract}


(109) 109

120

133

(This work) (109) TACCAGGACT TAT TAATA T T T A T T EU998743.4 (109) TACCAGGACTTATTAATATTTGATT AY228468.2 (109) TACCAGGACTTATTAATATTTGATT FJ899560.1 (109) TACCAGGACTTATTAATATTTGATT FJ899558.1 (109) TACCAGGACTTATTAATATTTGATT FJ899566.1 (109) TACCAGGACTTATTAATATTTGATT FJ899570.1 (109) TACCAGGACTTATTAATATTTGATT FJ899574.1 (109) TACCAGGACTTATTAATATTTGATT FJ899576.2 (109) TACCAGGACTTATTAATATTTGATT FJ899568.1 (109) TACCAGGACTTATTAATATTTGATT FJ899577.1 (109) TACCAGGACTTATTAATATTTGATT FJ899580.1 (109) TACCAGGACTTATTAATATTTGATT FJ899581.1 (109) TACCAGGACTTATTAATATTTGATT GQ478178.1 (109) TACCAGGACT TATTAATATTTGATT GQ478177.1 (109) TACCAGGACT TATTAATATTTGATT GQ478179.1 (109) TACCAGGAC T TA T TAATATT T GATT GQ478180.1 (109) TACCAGGACTTATTAATATTTGATT GQ478181.1 (109) TACCAGGACTTATTAATATTTGATT GQ478183.1 (109) TACCAGGACTTATTAATATTTGATT JN854153.1 (109) TACCAGGACTTATTAATATTTGATT JN854154.1 (109) TACCAGGACTTATTAATATTTGATT JN854159.1 (109) TACCAGGACTTATTAATATTTGAT T JN854168.1 (109) TACCAGGACTTATTAATATTTGATT JN854182.1 (109) TACCAGGACTTATTAATATTTGATT JN854211.1 (109) TACCAGGACTTATTAATATTTGATT JN854219.1 (109) TACCAGGACTTATTAATATTTGATT JN854226.1 (109) TACCAGGACTTATTAATATTTGATT KP099650.1 (109) TACCAGGACTTATTAATATTTGATT KP412541.1 (109) TACCAGGACT TATTAATATT TGATT KT723438.2(109) TACCAGGACTTATTAATATTTGATT KX255674.1 (109) TACCAGGACTTATTAATATT TGAT T FJ899555.2 (109) TACCAGGACTTATTAATATTTGATT FJ899561.2 (109) TACCAGGACTTATTAATATTTGATT FJ899563.2 (109) TACCAGGACTTATTAATATTTGATT FJ899564.2 (109) TACCAGGACTTATTAATATTTGATT FJ899569.1 (109) TACCAGGACTTATTAATATTTGATT FJ899575.1 (109) TACCAGGACTTATTAATATTTGATT JN854152.1 (109) TACCAGGACTTATTAATATTTGATT JN854160.1 (109) TACCAGGACTTATTAATATTTGATT JN854161.1 (109) TACCAGGACTTATTAATATTTGATT JN854163.1 (109) TACCAGGACTTATTAATATTTGATT JN854165.1 (109) TACCAGGACT TAT TAATATT T GATT JN854167.1 (109) TACCAGGACTTATTAATATTTGATT JN854171.1 (109) TACCAGGACTTATTAATATT TGAT T JN854172.1 (109) TACCAGGACTTATTAATATTTGATT JN854175.1 (109) TACCAGGACTTATTAATATTTGATT JN854176.1 (109) TACCAGGACTTATTAATATTTGATT JN854178.1 (109) TACCAGGACTTATTAATATTTGATT JN854177.1 (109) TACCAGGACTTATTAATATTTGATT JN854180.1 (109) TACCAGGACTTATTAATATTTGATT JN854183.1 (109) TACCAGGACTTATTAATATTTGATT JN854186.1 (109) TACCAGGACTTATTAATATTTGATT JN854187.1 (109) TACCAGGACTTATTAATATTTGATT JN854188.1 (109) TACCAGGACTTATTAATATTTGATT JN854189.1 (109) TACCAGGACTTATTAATATTTGATT JN854193.1 (109) TACCAGGACTTATTAATATTTGATT JN854196.1 (109) TACCAGGACTTATTAATATTTGATT
JN854198.1 (109) TACCAGGACTTATTAATATTTGATT JN854199.1 (109) TACCAGGACTTATTAATATTTGATT JN854201.1 (109) TACCAGGACTTATTAATATTTGATT JN854205.1 (109) TACCAGGACTTATTAATATTTGATT JN854202.1 (109) TACCAGGACTTATTAATATTTGATT JN854206.1 (109) TACCAGGACTTATTAATATTTGATT JN854208.1 (109) TACCAGGACTTATTAATATTTGATT JN854214.1 (109) TACCAGGACTTATTAATATTTGATT JN854215.1 (109) TACCAGGACTTATTAATATTTGATT JN854216.1 (109) TACCAGGACTTATTAATATTTGATT JN854218.1 (109) TACCAGGAC T TAT TAATAT T TGAT T JN854222.1 (109) TACCAGGACTTATTAATATTTGATT JN854225.1 (109) TACCAGGACTTATTAATATTTGATT KC427273.1 (109) TACCAGGACTTATTAATATTTGATT

D17510.1 (109) TACCAGGACTTATTAATATTGATT FJ899556.1 (109) TACCAGGACTTATTAATATTTGATT FJ899562.1 (109) TACCAGGACTTATTAATATTTGATT FJ899572.2 (109) TACCAGGACTTATTAATATTTGATT FJ899579.1 (109) TACCAGGACTTATTAATATTTGATT JN854151.1 (109) TACCAGGACTTATTAATATTTGATT JN854156.1 (109) TACCAGGACTTATTAATATTTGATT JN854158.1 (109) TACCAGGACTTATTAATATTTGATT JN854162.1 (109) TACCAGGACTTATTAATATTTGATT JN854173.1 (109) TACCAGGACTTATTAATATTTGATT JN854179.1 (109) TACCAGGACTTATTAATATTTGATT JN854181.1 (109) TACCAGGACTTATTAATATTTGATT JN854185.1 (109) TACCAGGACTTATTAATATTTGATT JN854190.1 (109) TACCAGGACTTATTAATATTTGATT JN854191.1 (109) TACCAGGACTTATTAATATTTGATT JN854194.1 (109) TACCAGGACTTATTAATATTTGATT JN854197.1 (109) TACCAGGACTTATTAATATTTGATT JN854200.1(109) TACCAGGACTTATTAATATTTGATT JN854209.1 (109) TACCAGGACTTATTAATATTTGATT JN854210.1 (109) TACCAGGACTTATTAATATTTGATT JN854224.1 (109) TACCAGGACTTATTAATATTTGATT KC427272.1(109) TACCAGGACTTATTAATATTTGATT KP771703.1 (109) TACCAGGACTTATTAATATTTGATT KR476379.1(109) TACCAGGACTTATTAATATTTGATT KT740995.1 (109) TACCAGGACTTATTAATATTTGATT KX833097.1(109) TACCAGGACTTATTAATATTTGATT EU998744.3 (109) TACCAGGACTTATTAATAT T TGATT EU998745.4(109) TACCAGGACTTATTAATATTTGATT EU998746.4 (109) TACCAGGACTTATTAATATTTGATT FJ899557.1 (109) TACCAGGACTTATTAATATTTGATT FJ899567.2 (109) TACCAGGACT TATTAATATTTGATT JN854164.1 (109) TACCAGGACTTATTAATATTTGATT JN854166.1 (109) TACCAGGACTTATTAATATTTGATT JN854174.1 (109) TACCAGGACTTATTAATATTTGATT JN854184.1 (109) TACCAGGACTTATTAATATTTGATT JN854192.1 (109) TACCAGGACTTATTAATATTTGATT JN854203.1(109) TACCAGGACTTATTAATATTTGATT JN854207.1 (109) TACCAGGACTTATTAATATTTGATT JN854213.1(109) TACCAGGACTTATTAATATTTGATT JN854220.1 (109) TACCAGGACTTATTAATATTTGATT EU998741.4(109) TACCAGGACTTATTAATATTTGATT EU998742.4 (109) TACCAGGACTTATTAATATTTGATT FJ899559.1 (109) TACCAGGACTTATTAATATTTGATT JN854223.1 (109) TACCAGGACTTATTAATATTTGATT KR873010.1(109) TACCAGGACTTATTAATATTTGATT 